\title{
Assessment of oral health knowledge, status and awareness among visually impaired children in Saudi Arabia
}

\section{Introduction}

Oral health and status have been associated with different parts of our daily life, including psychological, biological and social experiences as it is simply affected and influenced by aesthetics and communication. Having a good oral health is much significant in having a better appearance, mastication speech, health and digestion. Oral health could be related to a good general health in addition to being happy, as there are studies shown that esthetically unsatisfactory and functionally deficient dentitions might affect self-esteem, socialization and confidence. ${ }^{1}$ According to the World Health Organization, ${ }^{2} 19$ million children aged below 15 years are visually impaired. However, the prevalence of visual impairment worldwide has been decreasing since the early 1990s. That decrease was associated with different factors including
A) Socioeconomic development
B) Concerned public health actions
C) General population awareness. ${ }^{3}$

According to the WHO untreated cataract $(43 \%)$ is the most common cause of visual impairment after that comes diabetes mellitus $(24 \%){ }^{2}$ Some studies suggested that different diseases might be associated with visual impairment including other medical problems, such as cardiac defects or diabetes, which are more effective on dental care than the visual impairment. ${ }^{4}$ The oral health promotion relies mainly on oral health instructions, which it helps in prevention of the oral diseases especially in children as it assists in providing a good oral health through their whole life. ${ }^{5}$ In Saudi Arabia, the prevalence showed $1.5 \%$ of the population are visually impaired still, no sufficient studies regarding the subject were reported.

\section{Literature review}

\section{Methods of literature search and appraisal}

This research was conducted from October 2017-January 2018. It started with looking into previous articles (Full Articles) dated from (1965 - 2017) using different search engines and applications including Google Scholar, PubMed and ReadCube for relevant articles and reviewed their references list for additional citation using related keywords. All the researched articles were published in English language from different countries and journals around the world. The process of finding the right keywords was in the Inclusion of words such as (Blindness, Visually Impairment, Caries, Oral Hygiene...etc.) excluding articles having their samples from the age of 15 and above. This would help us keep our scope in the field of blindness/visually impairment within the selected age 6-15 years old in respect to their oral health status knowledge.

\section{Prevalence of visual impairment in Saudi Arabia}

Some studies have shown that the oral health status of the visually impaired population is notably worse than the sighted ones. It has

\author{
Volume 9 Issue 3 - 2018
}

\author{
Mustafa Alshehri,' Najla Alghamdi,' Mansour \\ Assery,' Abdulrahman Al Saffan,' 'Hoda \\ Abdellatif $^{2}$ \\ 'Department of Dentistry, Riyadh Elm University, Saudi Arabia \\ ${ }^{2}$ College of Dentistry, Princess Nourah University, Saudi Arabia
}

Correspondence: Mustafa Al Shehri, Department of Dentistry, Riyadh Elm University, Riyadh, Saudi Arabia, Tel 9665580000 40, Email Mustafa.Shehri@gmail.com

Received: June 01, 2018| Published: June 19, 2018

been reported that the visually impaired were far less knowledgeable about their oral health and hygiene status in comparison to the nonvisually impaired people. ${ }^{6}$ Another study done in China showed that $92 \%$ of the visually impaired weren't attending their regular dental visits. $85 \%$ were developing periodontal pockets $31 \%$ of them were deep pockets. ${ }^{7}$ Another point to look at is that visual impaired patients may not get hold on specific non-verbal aspects and might be on a disadvantage regarding the issue. The lack of dental clinics that provides Braille cards for appointments or information sheets. ${ }^{8}$ Some treatments might get invasive and become threatening, such scenarios with a visual impairment might make this more difficult, it is advised that commencing ideal treatment should be in short appointments until the patient is used to the treating dental staff. ${ }^{9}$

\section{Access to oral health care}

Supplying healthcare to the visually impaired is still facing many obstacles. Transportation difficulties, lack of social awareness, lack of training and educating of service providers and inadequate resources. ${ }^{10}$ Miller $\mathrm{S}^{11}$ stated the reluctant shown by the private practitioner might create a barrier in giving an ideal dental care to the disables population by providing negative contribution to the special patients rather than accommodating them. Some of the reasons that might cause these negative attitudes include; lacking in education and experience towards treating these patients, fear of going through treatment, unable to understand the physical condition of the patient, not trying to build a formidable relationship with the patient and unable to accept the substandard fees willingly. ${ }^{12}$

\section{Medical complications of visually impairment relevant to dentistry}

In a study done in 2016 showed that medical conditions associated with blindness might show an impact on the oral health status of the visually impaired children. The study was done on blind school children found that low vision or blindness had psychological implications including depression, sadness and anxiety. This may increase because of different feelings of inadequacy, facing difficulties in social interactions with people and making contacts. ${ }^{13}$ In a study done on the visually impaired children in Riyadh, Saudi Arabia 
showed more medical problems compared with their sighted peers. Factors such as poor OHI scores, higher PI scores, and higher GI scores were observed among the visually impaired children compared with the sighted group. ${ }^{14}$

\section{Communications}

Many individuals with a visual disability receive dental care in the General Dental Service and those with complicating medical conditions may be seen in community and hospital dental services. ${ }^{4}$ Freeman described the dentist to patient communications in four broad forms: verbal, non-verbal, affective/paralinguistic and written. ${ }^{15}$ Other studies of relevance have shown that individuals with a visual impairment are usually relying on verbal instructions and responding well to simple information which is perfectly explained. ${ }^{16} \mathrm{O}$ 'Donnell $\&$ Crosswaite ${ }^{5}$ found that blind children were very adept at converting oral instructions into performing oral hygiene practices. This will confirm that most given adequate verbal instructions to individuals with a visual impairment can have the same levels of oral health as their sighted peers. A good oral hygiene instruction can have a positive impact on oral hygiene, periodontal status and maintain or improve self-esteem. ${ }^{16}$

\section{Choice and use of dental services}

Watson $\mathrm{EK}^{10}$ conducted a study between two groups (visually impaired and non-visual impaired) the VI group unveiled more likely to request extraction of an aching posterior tooth or anterior tooth rather than seek restoration in comparison to the non-visually impaired group. ${ }^{10}$

\section{Preventive measures}

Advancing in the dental health education and following the preventive procedures will mostly demand different modifications Lange BM in 2013 Suggested that preventive procedures and home care should be taken seriously and be held along to be offered and adopted to the visually impaired population. Jain et.al stated that a great responsibility goes on to the dental health educators (Dentists, Assistants and Hygienists) to figure multiple ways in trying to help the visually impaired and the disabled in general, to hold on to these instructions and specifications. Dentists in general must evaluate carefully the abilities and disabilities of their patients', including the environment they're living upon and the people they're living with. Another issue that might be included is to provide discreptions and directions to the dental office and eliminate any obstacles or barriers in the office. Jain et al. also suggested that implementation of tooth brushing lessons in schools and teaching oral hygiene basics would reinforce and help disabled children to improve and promote good oral health.

\section{Oral hygiene education}

Dorout $^{17}$ implied that both type of blindness was often more knowledgeable about their own oral health status. ${ }^{17}$ However, in Taiwan, a study had established that the general knowledge of the oral health among visually impaired individuals were poor compared to their sighted peers. ${ }^{18}$ To fix this dilemma, action has to be taken in the field of the oral health education and instruction. The first step is to analyze and inspect the oral hygiene. The recommended method to the visually impaired is by educating them to use their fingers to locate each teeth position, and to have an idea of the differences in tooth anatomy, to place the toothbrush in the wanted area. A dentist can guide them by placing the child's hand on top of the dentist's in order to give a demonstration to them and feel the correct brushing motion. ${ }^{19}$ With the introduction of the electric tooth brush it was debatable whether it would be a help to the VIC. As in 2012 Sharma et, al. reported that electric tooth brushes have a huge effect in the prevention of plaque for the VIC. Hence another study done in 2011 suggested that there are no differences in effectiveness between using an electric toothbrush and using a manual toothbrush. ${ }^{20}$ One study found that using the oral hygiene educating method has no effect upon the visually impaired population. ${ }^{21}$ As there are some instances where visual aids should be portrayed (eg. Disclosing Agent Tablets) are not appropriate to be demonstrated in front of the visually impaired population. ${ }^{5}$ However, it is unfortunate to say that little information is being researched and found online on such population here in Saudi Arabia. ${ }^{17}$

\section{Oral hyigene instructions (plaque removal techniques)}

In 2016 a study done by Reshma Mohan ${ }^{22}$ implied that brushing instruction should begin with the patient demonstrating his / her current brushing technique, which can further be corrected by verbal and physical guidance. Fone's technique and Modified Bass technique of tooth brushing were suggested to be very effective in providing a good oral hygiene of the visually impaired. But, for most people, the horizontal method looked to offer a better result to other techniques due to the easiness of application. Soft bristled, multi-tufted nylon brushes with rounded tips are advised. The size of the brush should be appropriate for the individual's mouth. Time is an important factor in the effectiveness of brushing method. Children who brush at least three minutes are more effective in removing plaque than children who brushed for a shorter time. Using electric toothbrush may also help the visually impaired children compared to a manual toothbrush. A specific sequence of brushing should be established to make sure that all the areas of the mouth are brushed adequately. Musical tunes or songs are available to teach the basic steps to children with visual impairment as visually impaired depend much more on hearing and touch for proper orientation. The use of floss for plaque removal is important, if the caretaker is performing the flossing. However, flossing can be made easier if a floss holder is used. The support of tactile sensation would help in the placement of the brush or floss in the correct position inside the mouth. ${ }^{22}$

\section{Parent's responsibilities}

Visually impaired children usually face difficulty in achieving an adequate tooth brushing procedure; this includes the application of the tooth paste on the toothbrush as well as the performance. These strains would be demonstrated by oral hygiene supervision. ${ }^{17}$ Furthermore, the lack of knowledge and behavior in oral health by the parents by not getting their children to a regular dental checkup would not help ease the problem of the progression of a poor oral health among the visually impaired children. Manually developing these dexterities for the visually impaired children would allow for a complete control of the process independently without the help of a secondary hand. ${ }^{17}$

\section{Social involvement}

In a recent study ${ }^{8}$ conducted in Saudi Arabia showed that the visually impaired population has a poor plaque control, gingivitis and dental caries, as well as the limitation and difficulties for accessing the health care providers. As they need extensive oral health promotion 
programs control plaque and prevent caries. Visual impairment may impact on oral health through physical social or information barrier related to the impairment, attendant medical conditions or lack information in a suitable format. Other difficulties include the lack of transport and services, insufficient resources or financial considerations, lack of social awareness, education and the training of service provider employees. ${ }^{23}$

\section{Purpose of this study}

The purpose of this study is to evaluate the oral health condition within the visually impaired children aged 6-15 in the city of Riyadh, Saudi Arabia.

\section{Specific objectives}

1. Assess oral health knowledge among school children in Riyadh

2. Estimate the distribution and severity of major oral diseases and conditions among

3. Estimate the need for care and need for community-oriented disease prevention programs for this selected population

Estimate the nature and urgency of the oral health treatment(s) required in this population.

\section{Methods}

The oral health survey of the visually impaired subjects was held in Riyadh Elm University (REU) -Namuthajiah Campus- in Riyadh. The subjects of this study were within the age of 6-15 years old.

It consists of two components, oral health examination and an oral health survey questionnaire. The following paragraphs describe the methods and study design used for each component and the study, overall.

\section{Definition of visual impairment}

According to the 10 th revision of the $\mathrm{WHO}^{2}$ International Statistical Classification of Diseases, Injuries and Causes of Death, 'Low Vision' is defined as visual acuity of less than $6 / 18$ but equal to or better than $3 / 60$, or a corresponding visual field loss to less than $20^{\circ}$, in the better eye with the best possible correction. 'Blindness' is defined as visual acuity of less than $3 / 60$, or a corresponding visual field loss to less than $10^{\circ}$, in the better eye with the best possible correction. 'Visual impairment' includes both low vision and blindness. Which we targeted in our research.

\section{Study design}

The Survey of the Oral Health Status of Riyadh of visually impaired children employed a multi-stage sample selection. The first stage involved getting a list of all public schools for visually impaired in Riyadh city by the Ministry of Education from which sampling will be carried out. The second stage, letter of introduction was sent to 16 schools in Riyadh. The purpose of the letter was to introduce the study, request permission to conduct the screening and to schedule visits to the clinics of dental school of Riyadh Elm University (Namuthajiah Campus). Once the school directors agreed to participate, arrangement of the school visit date was made. The third stage involved obtaining agreement of all children who are enrolled in the 16 schools to participate in the study in a total of 147 male students and 120 female students. 16-school 120-female 147-male.

\section{Dental examination}

The dental examination was conducted into sites, one at REU dental clinics and at the school sites.

REU Dental Clinics: The oral health examinations were conducted at the REU dental clinics in two days. Subjects were examined seated in the dental chair, and the examiner assisted by a recorder (dental intern) familiar with the examination coding criteria. The examination was done under LED lighting from the dental chair and used dental examination kits (1 Mouth Mirror, 1 Tweezer, 1 Dental Explorer and 1 WHO Dental Probe). Infection control and waste disposal recommendations and standards were followed. Once the examination is completed, each child received oral hygiene instruction using jaw model. A copy of the screening report was sent to the parents with the child treatment needs results with a list of referral oral health care facilities. It started with hosting the samples in REU for two successive days with the aid of 15 dental interns. They were first taken to a room to be interviewed by dental interns using the WHO Oral Health Questionnaire for Children, after completion they were taken for the dental examination. REU helped by providing two fixed dental chairs (Sirona) to the study site, as well as dental examination kits and jaw models. The examination started by taking samples to the dental chair, a dental intern assisted the examiner by documenting the WHO's oral health assessment form for children. While two samples were examined the others were held with the dental interns demonstrating and educating their oral health by using brushing techniques, flossing and sensing tooth surfaces on jaw models given. The total samples collected in the 2 days were 112 males and 91 females.

\section{Data collection}

Data collection from the subjects participating in the study will be consisted of two parts - an oral health questionnaire, and an oral health examination. The WHO oral health assessment form and oral health questionnaire for children will be used

Oral health questionnaire: The first part is questionnaire to the student, designed to collect demographic characteristics and oral health knowledge and attitude. The questionnaire will be translated to Arabic and back translated to English by persons well versed with both the languages. The WHO questionnaire will be administered face to face by REU dental interns. A calibration and training for the dental interns was done before the start of the interview to the visually impaired children.

Oral health examination: The second part is the oral health examination, which will include assessments of dentition status, gingival status, enamel fluorosis, dental erosion, dental trauma, oral mucosal lesions, and intervention urgency. Training and calibrating examiner was conducted to ensure uniform interpretation, understanding and application by examiner of the criteria and codes for the various diseases and conditions to be observed and recorded, and that each examiner can examine consistently. Intra- examiner reliability was assessed.

\section{Sample size and study population}

The sample of this study is a convenient sample of students from 16 schools agreed to participate in the study. 


\section{Ethical considerations}

In order for a child to participate in the screening, a signed consent was required. The consent form included an introduction to the oral health survey that was sent to the parents/guardians of the schoolchildren. After examination, a report card was given to the child's parent with regard to the child treatment needs assessment.

\section{Results}

Our sample was summed up in 148 males and 119 female individuals from different primary and middle schools (aged 6-15) in Riyadh, Saudi Arabia. The sample were given the questionnaire and interviewed following the WHO oral health assessment form for children. Most of the male and female children were aged 11 years old (Table 1). Upon interviewing, the overall results came out showing interesting answers. In the question that was asking "How would you describe the health of your teeth?", most answers came out positive, $40 \%$ of the males and $57.5 \%$ of the females answered "Excellent" (Table 2). While in the question that asked "How often during the past 12 months did you have a toothache or feel discomfort due your teeth?"; "Occasionally" was the answer for genders, $30.6 \%$ in males and $26.7 \%$ in females (Figure 1). Another interesting answer was shown in the question of "How often did you go to the dentist during the past 12 months?", "I have never received a dental care from a dentist" was the answer of choice for both genders followed by "Once". As $36.7 \%$ of the males and $28.3 \%$ of the females had the former answer, while $19 \%$ of males and $27.5 \%$ of females had the latter. The next question followed by "What was the reason for your last visit to the dentist?" The most answer of both genders was "Pain or trouble with teeth, gums or mouth" in a total of 58.5\% (Figure 2). Another question showed that $63.3 \%$ of females tend to avoid smiling and laughing in the public because of their status of their teeth. And when the samples were asked regarding their parents' level of education, most of their mothers haven't completed their primary school education.

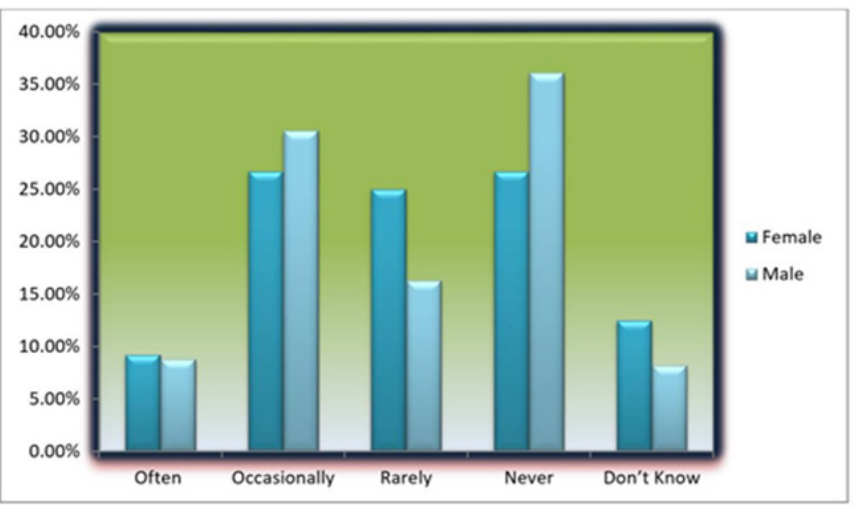

Figure I Rate of toothache or feel discomfort at the last year. $(\mathrm{N}=269)$.

\section{Discussion}

Visually impaired children are facing challenges and obstacles everyday due to their disabilities. Different studies have related the negligence to the people carrying disabilities by their families and the surrounding society when it comes to general health. This study was focusing on the oral health and has shown how much care and
Table I Socio-demographic characteristics of the study sample $(\mathrm{N}=269)$

\begin{tabular}{lllll}
\hline \multirow{2}{*}{ Varibles } & \multicolumn{4}{l}{ The study smaple $(\mathbf{n = 2 6 9 )}$} \\
\cline { 2 - 5 } & Female (120) & $\%$ & Male & $\%$ \\
\hline Age years: & & 49.2 & 75 & 51.1 \\
$06-10$ & 59 & 50.8 & 72 & 48.9 \\
II-15 & 61 & & $7-14$ & \\
Range & $7-15$ & & $9.78 \pm 2.15$ \\
Mean $\pm S D$ & $9.91 \pm 2.41$ & &
\end{tabular}

Table 2 Opinion of the study sample about their gum \& tooth health $(\mathrm{N}=269)$

\begin{tabular}{lllll}
\hline \multirow{2}{*}{ Teeth \& gum health } & \multicolumn{4}{l}{ The study sample } \\
\cline { 2 - 5 } & Female & $\%$ & Male & $\%$ \\
\hline Teeth's health & 69 & 57.5 & 59 & 40.1 \\
Excellent & 15 & 12.5 & 45 & 30.6 \\
very good & 3 & 2.5 & 15 & 10.2 \\
good & 18 & 15 & 11 & 7.5 \\
average & 1 & 0.8 & 10 & 6.8 \\
poor & 14 & 11.7 & 7 & 4.8 \\
Don't know & & & & \\
Gum's health & 62 & 51.7 & 72 & 49 \\
Excellent & 18 & 15 & 40 & 27.2 \\
very good & 4 & 3.3 & 9 & 6.1 \\
good & 13 & 12.5 & 11 & 7.5 \\
average & 75.8 & 8 & 5.4 \\
poor & & 10.8 & 7 & 4.8 \\
Don't know & & & & \\
\hline
\end{tabular}

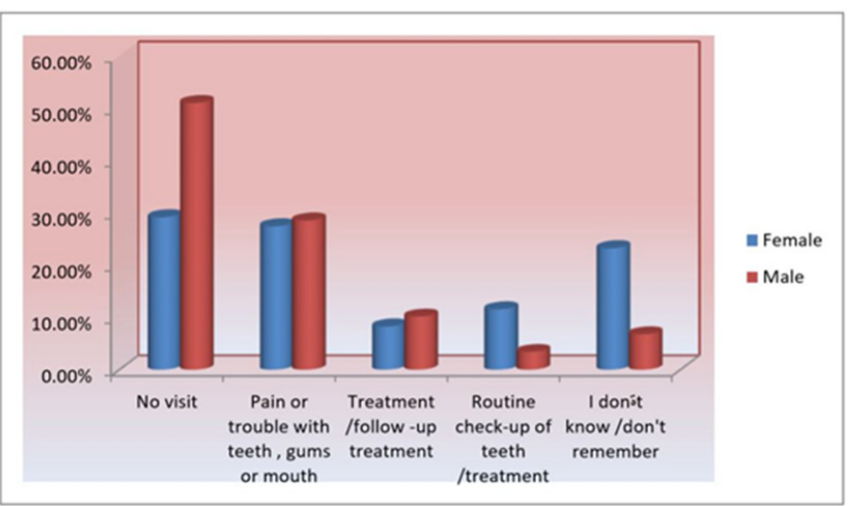

Figure 2 The reason for your last visit to the dentist at the last year $(\mathrm{N}=269)$

attention the visually impaired children should get. The carelessness is shown with the related answers of the WHO health assessment form for children, in questions such as "How often during the past 12 months did you have a toothache or feel discomfort in your teeth?" (Figure 1) and "How often did you go to the dentist during the past 12 months?" (Figure 2) it shows how they've been only visiting the dentist for an emergency reason once in the whole year while the 
majority haven't been in a dental clinic at all. Another study done in 2013 regarding the same subject in Saudi Arabia showed more than $50 \%$ indicated symptomatic attendance as $20 \%$ have never been in a dental clinic. ${ }^{23}$ Regular dental checkups and routine dental examinations were critically missing, as that would help prevent a lot of further complications. Social complications are also presented in this study as well, showing in the question that asked "Do you avoid smiling and laughing because of your teeth" where female children are facing the bigger part of it, shown and reflected by their positive answers to this question. Mahoney EK ${ }^{4}$ indicated that Self-modeling could efficiently be used in providing a good dental health instruction, as well as a full consideration of their impairment. Their parents of course share the majority of their problems as their guardians. But still some of the families weren't enough educated to give the better picture of a healthy oral health to their children. It's a shared problem everyone should take a part in responsibility. Vignesha H stated that the high prevalence of dental problems in disabled children seem to be related to the poor use of dental services and the lack in dental awareness. Improving the access to different dental services as well as the implementation of oral health education is a necessity to ensure that optimum oral health is reachable of these less fortunate children (Vignesha H. 1991).

\section{Conclusion and recommendation}

Proper public health education is critically required in schools or in public areas; it should be directed to the children and their parents as well. This would help in creating a better well-educated generation, which would help in a better understanding of proper prevention methods within the society which will then eventually be brought to every house. ${ }^{24-42}$

\section{Acknowledgements}

The Completion of this undertaking could not have been possible without the participation and assistance of so many people, in particular; my parents, my dear wife Dr. Najla Alghamdi for her love and support and our families for their endless contribution. As well as Prof. Hoda Abdullatif for her measureless aid and supervision, our program director Dr. Navin Ingle for his assistance and REU.

\section{Conflict of interest}

Author declares that there is no conflict of interest.

\section{References}

1. Fiske J, Davis DM, Frances C, et al. The emotional effects of tooth loss in edentulous people. Br Dent J. 1998;184:90-93.

2. World Health Organization. World Health Report 1998; 2004.

3. Bourne RRA, Flaxman SR, Braithwaite T, et al. Magnitude, tempora trends, and projections of the global prevalence of blindness and distance and near vision impairment: a systematic review and meta-analysis. Lancet Glob Health. 2017;5(9):e888-e897.

4. EK Mahoney, N Kumar, SR Porter. Effect of visual impairment upon oral health care: a review. Br Dent J. 2008;204(2)63-7.

5. O Donnell D, Crosswaite MA. Dental Health Education for the Visually Impaired Child. J Royal Soc Promot Health. 1990;110(2):60-61.

6. Nandini NS. New insights into improving the oral health of visually impaired children. J Indian Soc Pedod Prev Dent. 2003;21(4):142-143.
7. Chang CS, Shih Y. Teaching Oral Hygiene Skills to Elementary Students with Visual Impairments. J Vis Impair Blind. 2005;91(1):26-39.

8. Edwards DM, Merry AJ. Disability part 2: access to dental services for disabled people. A questionnaire survey of dental practices in Merseyside. Br Dent J. 2002;193(5):253-255.

9. Davis RL. The blind dental patient. Ill Dent J. 1965;34:18-21.

10. Watson EK, Moles DR, Kumar N. Online article number E15 Refereed Paper-accepted 19 February 2010. Br Dent J. 2010; 208:E15.

11. Miller S, Heil J. Effect of an extramural programm of dental care for the special patient on attitudes of students. J Dent Educ. 1976;40(11):740-44.

12. Wessels KE. Dentistry for the handicapped patient. $77-110$.

13. Ishtiaq R, Chaudhary MH, Rana MA, et al. Psychosocial implications of blindness and low vision in students of a school for children with blindness. Pak J Med Sci. 2016;32(2):431-4.

14. Al Sadhan, Asma M Al Jobair, Mariam Bafaqeeh, et al. Dental and medical health status and oral health knowledge among visually impaired and sighted female schoolchildren in Riyadh: a comparative study. $B M C$ Oral Health. 2017;17:154

15. Freeman R. Communication, body language and dental anxiety. Dent Update. 1992;19(7):307-309.

16. Schembri A, Fiske J. The implications of visual impairment in an elderly population in recognizing oral disease and maintaining oral health. Spec Care Dent 2001;21(6):222-226.

17. Ismail A Dorout. Knowledge and oral health related behavior among visually impaired subjects in Jazan Region, Kingdom of Saudi Arabia. $J$ Dent and Oral Hygiene. 2015;7(3):33-39.

18. Ng KKW, Wong MCM, Cheung MMK. Dental care for visually impaired institutionalized older persons in Hong Kong Community Health Project Report Series. Faculty of Dentistry, University of Hong Kong; 2003.

19. Lebowitz EJ. An introduction to dentistry for the blind. Dent Clin North Am. 1974;18:651-69.

20. Smutkeeree A, Rojlakkanawong N, Yimcharoen V. A 6-month comparison of toothbrushing efficacy between the horizontal scrub and modified Bass methods in visually impaired students. Int J Paedia Dent. 2011;21(4):278-83.

21. Scardina GA, Buonamente A, Messina P. Efficacy of an oral health training programme for visually impaired individuals. Ig Sanita Pubbl. 2008;64:509-16.

22. Mohan R. Comprehensive Dental Care for the Visually Impaired: a review. International Journal of Oral Health and Medical Research. 2016;3(4):97-101.

23. Aljoharah AA. Oral hygiene practices and periodontal health status of visually impaired Saudi adults in Riyadh, Saudi Arabia. Pak Oral Dent J. 2013;33(1):82-86.

24. Al-Qahtani Z, Wyne AH. Caries experience and oral hygiene status of blind, deaf and mentally retarded female children in Riyadh, Saudi Arabia. Odontostomatol Trop. 2004;27(105):37-40.

25. Bhat N, Agrawal A, Nagrajappa R. Teeth fracture among visually impaired and sighted children of 12 and 15 years age groups of Udaipur city, India - a comparative study. Dental Traumatology. 2011;27(5):389-392.

26. Becker A, Chaushu S, Shapira J. Orthodontic treatment for the special needs child. Seminars in orthodontics. 2004;10(4):281-292.

27. Gabre P, Martinsson T, Gahnberg L. Incidence and reasons for tooth mortality among mentally retarded adults during a 10-year period. Acta Odontologica Scandinavica. 1999;57(1):55-61. 
28. Greeley CB, Goldstein PA, Forrester DJ. Oral manifestations in a group of blind students. ASDC J Dent Child. 1976;43(1):39-41.

29. Jokic NI, Majstorovic M, Bakarcic D, et al. Dental caries in disabled children. Coll Antropol. 2007;31(1):321-4.

30. Kaneko N, Yoshihara A, Ida H, et al. Influence of a fluoride mouthrinse on mutans streptococci in school children. Caries Res. 2006;40(6):501-7.

31. Koseki M, Maki Y, Matsukubo T, et al. Salivary flow and its relationship to oral signs and symptoms in patients with dry eyes. Oral Dis. 2004;10(2):75-80.

32. Lawrence HP, Garcia RI, Essick GK, et al. A longitudinal study of the association between tooth loss and age related hearing loss. Spec Care Dent. 2001;21(4):129-140.

33. Loe H. Oral hygiene in the prevention of caries and periodontal disease. Int Dent J. 2000;50(3):129-139.

34. Mann J, Wolnerman JS, Lavie G. Periodontal treatment needs and ora hygiene for institutionalized individuals with handicapping condition. Spec Care Dentist. 1984;4(4):173-176.

35. Oliveira A, Paiva S, Martins MT. Prevalence and determinan factors of malocclusion in children with special needs. Eur J Orthod 2011;33(4):413-8.
36. Rapp R, Kanar HL, Nagler B. Pedodontic care for the deaf and blind Dent Clin North Am. 1966;10:21-34.

37. Royal National Institute for the Blind; 2004.

38. Scully C, Diz Dios P, Kumar N. Special care in dentistry-handbook of oral healthcare. London: Churchill Livingstone; 2006:1-512.

39. Magnitude, temporal trends, and projections of the global prevalence of blindness and distance and near vision impairment: a systematic review and meta-analysis. Lancet Glob Health. 2017;5(9):e888-e897.

40. Varghese RK, Agrawal A, Mitra A. Anterior teeth fracture among visually impaired individuals. India Journal of Advanced Dental Research. 2011;2(3):43-49.

41. Winter K, Baccaglini L, Tomar S. A review of malocclusion among individuals with mental and physical disabilities. Spec Care Dentist. 2008;28(1):19-26.

42. World Health Organization. Global Data on Visual Impairments 2010 2012 . 